\title{
Estimation of scattered radiation in digital breast tomosynthesis
}

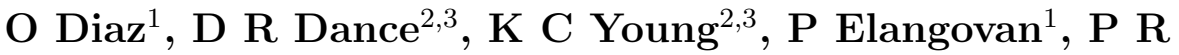 \\ Bakic $^{4}$ and K Wells ${ }^{1}$ \\ ${ }^{1}$ Centre for Vision, Speech and Signal Processing, University of Surrey, Guildford, \\ GU2 7XH, UK \\ ${ }^{2}$ National Coordinating Centre for the Physics of Mammography, Royal Surrey \\ County Hospital, Guildford, GU2 7XX, UK \\ ${ }^{3}$ Department of Physics, Faculty of Engineering and Physical Sciences, University of \\ Surrey, Guildford, GU2 7XH, UK \\ ${ }^{4}$ Department of Radiology, University of Pennsylvania, Philadelphia, PA 19104, USA \\ E-mail: k.wells@surrey.ac.uk
}

\begin{abstract}
.
Digital breast tomosynthesis (DBT) is a promising technique to overcome the tissue superposition limitations found in planar 2D X-ray mammography. However, as most DBT systems do not employ an anti-scatter grid, the levels of scattered radiation recorded within the image receptor are significantly higher than that observed in planar 2D X-ray mammography. Knowledge of this field is necessary as part of any correction scheme and for computer modelling and optimisation of this examination. Monte Carlo (MC) simulations are often used for this purpose, however they are computationally expensive and a more rapid method of calculation is desirable. This issue is addressed in this work by the development of a fast kernel-based methodology for scatter field estimation using a detailed realistic DBT geometry. Thickness-dependent scatter kernels, which were validated against the literature with a maximum discrepancy of $4 \%$ for an idealised geometry, have been calculated and a new physical parameter (air gap distance) was used to estimate more accurately the distribution of scattered radiation for a series of anthropomorphic breast phantom models. The proposed methodology considers, for the first time, the effects of scattered radiation from the compression paddle and breast support plate, which can represent more than $30 \%$ of the total scattered radiation recorded within the image receptor. The results show that the scatter field estimator can calculate scattered radiation images in an average of 80 minutes for projection angles up to $25^{\circ}$ with equal to or less than a $10 \%$ error across most of the breast area when compared with direct MC simulations.
\end{abstract}

PACS numbers: 87.10.Rt(MC simulations), 87.57.rh (Mammography), 87.59.ej(Digital mammography)

Submitted to: Phys. Med. Biol. 


\section{Introduction}

Breast cancer is the second most commonly diagnosed cancer worldwide (Ferlay et al. 2010). In order to reduce cancer mortality, early cancer detection represents a key factor which has motivated the establishment of national screening programmes in many Western countries. The standard radiological technique used in breast cancer screening is 2D planar X-ray mammography. Nevertheless, this technique suffers from limitations due to the superposition of 3D anatomical structures onto the 2D projected image (Chen \& Ning 2003). This superposition effect may obscure real lesions or even simulate the appearance of pathology, where none exists.

By contrast, digital breast tomosynthesis (DBT) (Kolitsi et al. 1992, Niklason et al. 1997, Wallis et al. 2012, Skaane et al. 2013) is an emerging technology which can overcome the aforementioned limitations (Dobbins \& Godfrey 2003). DBT uses a series of 2D low-dose X-ray projections of the breast taken at different (but limited) angles to create a pseudo-3D image representation of the breast. Therefore, visualisation of the breast at different depths is available, thus removing much of the obfuscating anatomical clutter.

One of the main disadvantages of using DBT is the significantly increased amount of scattered radiation observed in the image receptor for each projection compared with planar X-ray mammography. This is due to the absence of an anti-scatter grid in most of the DBT commercial systems. Generally, the image receptor remains static while the $\mathrm{X}$-ray tube is swept through a limited arc; thus a conventional fixed anti-scatter grid would absorb much of the primary photons as described by Sechopoulos et al. (2007 b). Therefore relatively large scatter fields are present in the projection images which need to be estimated as part of any correction scheme to partly compensate for the contrastreducing effect of this undesirable component, and to minimise the associated error during reconstruction ( $\mathrm{Wu}$ et al. 2009). Knowledge of this field is also required for performance and optimisation studies using simulation.

Monte Carlo (MC) simulation is a common technique to directly estimate scatter fields (Dance \& Day 1984, Boone \& Cooper 2000, Sechopoulos et al. 2007b, Cunha et al. 2010), however this can be extremely time-consuming, and calculation times of more than 10 hours have been reported by Diaz et al. (2012). Thus fast convolution-based methods using appropriate scatter kernels represent an attractive option to estimate scatter fields (Love \& Kruger 1987, Seibert \& Boone 1988, Boone \& Cooper 2000, Wu et al. 2009, Ducote \& Molloi 2010), albeit with limitations. One of the principal limitations of prior work has been the highly idealised geometries of both imaging system and object simulated.

By contrast, the convolution-based approach presented here for estimating scattered radiation distributions takes into account the full geometry found in a realistic DBT scenario, which includes the compression paddle and breast support. This has also facilitated a detailed study of the effects of the air gap between the lower curvature of the breast and the image receptor (Diaz et al. 2012), which has provided insight into 
the local and global variation of scatter across the image.

In the next section, a kernel-based methodology is described to calculate the scattered radiation. The proposed technique includes the use of anthropomorphic breast models integrated in a realistic DBT geometry. The results, shown in section 3, illustrate that the proposed scatter kernels can estimate scatter fields more accurately than using the scatter kernels previously described in the literature. With computational time more than five times faster than direct MC simulations, the proposed scatter-kernel method shows errors equal to or less than $10 \%$ across most of the breast phantom area when compared with MC-based results.

\section{Methodology}

The energy recorded within a DBT image receptor corresponds to primary and scattered $\mathrm{X}$-ray photons. In this work, analytically calculated primary radiation images were used to produce the scattered radiation images using a modified convolution. This included the use of a set of scatter kernels, which was constructed from previously calculated scatter point spread functions (SPSFs).

\subsection{Digital breast tomosynthesis geometry}

The DBT geometry used in this study approximately corresponds to the Hologic Selenia Dimensions system as illustrated in Figure 1. The X-ray tube, which was simulated as a point source, was placed at $700 \mathrm{~mm}$ from the detector surface (above the chest-wall side of field) for the $0^{\circ} \mathrm{X}$-ray tube position and the rotation axis was located across the middle of the image receptor. The configuration also contains, from top to bottom, a $2.8 \mathrm{~mm}$ thick compression paddle of polycarbonate, an anthropomorphic breast model which is described in section 2.3, a carbon-fibre breast support plate with a thickness of $1 \mathrm{~mm}$ and a $200 \mu \mathrm{m}$ a-Se detector with cover. The detector cover is located at a fixed distance of $24 \mathrm{~mm}$ from the bottom of the breast support. Despite the fact that the widest projection angle of this commercial system is $7.5^{\circ}$ in screening mode, projection angles up to $25^{\circ}$ have been studied as shown in Figure 1(a). This is in order to consider the effects of the wider angle range employed by other manufacturers (Baker \& Lo 2011, Sechopoulos 2013). In all cases studied, the X-ray beam was collimated to match the dimension of the image detector, which was sufficiently large to image the entire breast model in one projection. Thus, a 30x24 $\mathrm{cm}^{2}$ image receptor was used for imaging the 22 and $50 \mathrm{~mm}$ thick breast phantoms, whereas the dimension of the image detector for the the $77 \mathrm{~mm}$ thick breast phantom was $45 \times 24 \mathrm{~cm}^{2}$.

This geometry was used to calculate ground truth data using MC simulation, with which results from the proposed convolution-based methodology have been compared. 


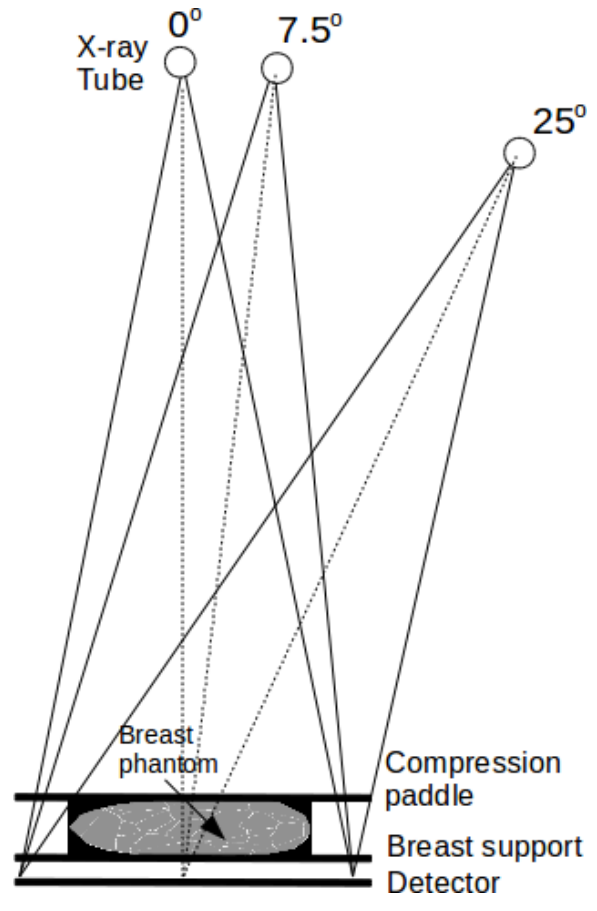

(a) Side view

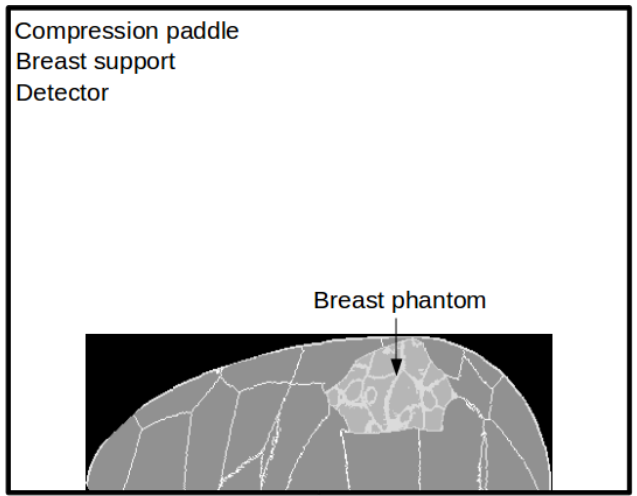

(b) Top view

Figure 1. DBT geometry: (a) side and (b) top view. The dimensions of the compression paddle and breast support plate match the detector size as show in (b) . These are illustrated using a rectangular region.

\subsection{Monte Carlo $(M C)$ simulation software}

In this study, MC simulations based on the GEANT4 toolkit $\ddagger$ (Agostinelli et al. 2003, Allison et al. 2006) were used to calculate primary and scatter fields. This MC package has been used successfully elsewhere in the X ray mammography fieldThis MC code has been previously validated against published data and experimentally acquired date (Diaz 2013). Results illustrated overall errors of 3\% in contrast improvement factor and Bucky factor values, and $4 \%$ in SPR values when comparing with Boone et al. (2002). Also, 1.5\% discrepancy in contrast was observed when comparing images acquired experimentally and images from MC. SPSFs were generated using narrow pencil beams to provide the appropriate scatter kernels.

The polyenergetic spectra used in the MC simulations were based on the calculated spectral data of Boone et al. (1997) and the experimental half value layer (HVL) was matched by the addition of an aluminium filter. In order to make the simulations realistic, the most clinically relevant energy spectrum for each breast phantom thickness was used. Thus $26 \mathrm{kVp} \mathrm{W} / \mathrm{Al}(\mathrm{HVL}=0.44 \mathrm{~mm} \mathrm{Al}), 31 \mathrm{kVp} \mathrm{W} / \mathrm{Al}(\mathrm{HVL}=0.54 \mathrm{~mm}$ $\mathrm{Al})$ and $36 \mathrm{kVp} \mathrm{W} / \mathrm{Al}(\mathrm{HVL}=0.63 \mathrm{~mm} \mathrm{Al})$ anode/filter combinations were used for the anthropomorphic breast phantom thicknesses studied (22, 50 and $77 \mathrm{~mm}$ ).

In the MC model, when a photon exits the point source X-ray tube, it is tracked

$\ddagger$ GEANT4 9.3.p02 
until it is completely absorbed or it leaves the system. During its passage through the imaging configuration, a photon is considered part of the primary beam unless it undergoes a scatter interaction (incoherent and/or coherent), in which case the photon is tagged as scatter.

When a photon reaches the image receptor, the energy deposited by incoherent scattering and photoelectric interactions as well as their spatial locations are stored and used to create the accumulated image after the simulation.

The imaging configuration described in section 2.1 was inserted in the MC code and 10 runs of $10^{9} \mathrm{X}$-ray photons were simulated for each case considered, irradiating the entire image receptor using a pixel size of $1 \mathrm{~mm}^{2}$. As the scatter represents a low frequency signal, a $7 \times 7$ median filter was applied five times in order to smooth the the MC calculated scatter image, showing a maximum statistical error of $0.25 \%$ (standard error of the mean, SEM) when the images from the 10 runs were combined.

\subsection{Anthropomorphic breast phantom}

Anthropomorphic breast phantoms developed by Bakic et al. (2011) were used in this study to compare the direct MC and other methods of estimating primary and scatter fields. These compressed breast phantoms contain simulated tissue structures found in real breasts as follows: adipose tissue, glandular tissue and Cooper's ligaments surrounding by a layer of skin. The compositions of the adipose tissue, glandular tissue and skin within the breast phantom were taken from Hammerstein et al. (1979), whereas the composition for Cooper's ligaments has been assumed to be that of adult skeletal muscle (ICRU 1992). Three breast phantoms have been used in this study, whose parameters are given in Table 1 . Note that a relatively large voxel size $(0.5 \times 0.5 \times 0.5$ $\mathrm{mm}^{3}$ ) was used to accelerate the MC calculations.

Table 1. Parameters of the breast phantoms used in this study.

\begin{tabular}{ccccc}
$\begin{array}{c}\text { Thickness } \\
(\mathrm{mm})\end{array}$ & $\begin{array}{c}\text { Glandularity } \\
(\%)\end{array}$ & $\begin{array}{c}\text { Voxel size } \\
\left(\mathrm{mm}^{3}\right)\end{array}$ & $\begin{array}{c}\text { Dimension } \\
\left(\mathrm{mm}^{2}\right)\end{array}$ & $\begin{array}{c}\text { Area } \\
\left(\mathrm{cm}^{2}\right)\end{array}$ \\
\hline 22 & 30 & & $174.5 \times 49.0$ & 65.1 \\
50 & 20 & $0.5 \times 0.5 \times 0.5$ & $204.5 \times 65.0$ & 100.1 \\
77 & 19 & & $307.0 \times 94.5$ & 221.0
\end{tabular}

\subsection{Primary transmission calculation}

The image generated from the primary (i.e. unscattered) beam $P(x, y)$ observed across each pixel $(x, y)$ at the image receptor was calculated analytically using the BeerLambert's law:

$$
P(x, y)=\sum_{E=E_{\min }}^{E_{\max }} \epsilon(E) \cdot w(E) \cdot E \cdot e^{\sum_{i}\left(-\mu_{i}(E) \cdot t_{i}(x, y)\right)} \quad[e V],
$$

where $\mu_{i}(E)$ represents the linear attenuation coefficients of the different $i$ tissues found along the photon path $t_{i}(x, y)$. Each pixel at the image receptor is evaluated 
1

at energy intervals, $d E$, across the complete energy spectrum $w(E)$ emitted from the $\mathrm{X}$-ray tube. The energy-dependent absorption efficiency of the image receptor, which was calculated separately using MC simulations, is denoted by $\epsilon(E)$.

The photon path lengths $t_{i}(x, y)$ through material $i$ in the voxelised breast phantom were computed using ray tracing (Siddon 1985), along a ray cast from the X-ray focus to the centre of each pixel in the image detector. In principle, the primary radiation needs to be averaged within the entire pixel area. For this reason, MC-based primary radiation, which sampled several points within the pixel area, was calculated and compared with the analytically calculated primary, which was calculated only at the centre of each pixel. Furthermore, since the X-ray focus is considered as a point source, neither focal spot blur nor motion blur effects were simulated.

\subsection{Scatter point spread functions (SPSFs)}

Narrow pencil beam methodology was used within the MC simulation to calculate the SPSF. This approach consisted of inserting a narrow pencil X-ray beam in a given imaging geometry, thus the scattered radiation recorded within the image receptor provides the scatter response function for the geometry considered.

Two pencil beam geometries, simulating the geometry found in the Hologic Selenia Dimension system (section 2.1.), were used. In the first setup (Figure 2(a)), a series of circular uniform phantoms with different thickness $(T)$, glandularity $(G)$, projection angle $(\phi)$ and air gap $(A G)$ between the lower surface of the uniform phantom and the breast support were used. The $T$ range was from 0.1 to $10 \mathrm{~cm}$ whereas only $G$ values of $0 \%$ and $100 \%$ were used. Also, $A G$ up to $50 \mathrm{~mm}$ in steps of $2 \mathrm{~mm}$ and $\phi$ of $0,7.5$ and $25^{\circ}$ were simulated. The pencil beams hit the centre of the circular phantoms and this was used to calculate the scatter response function within the breast shadow on the image receptor $\left(\mathrm{SPSF}_{b r e}\right)$ (i.e. breast phantom region).

As described by Dance \& Day (1984) and Sechopoulos et al. (2007b), scattered radiation from the components of the system (mainly compression paddle and breast support plate) contributes significantly to the scatter observed under the breast, especially near its edges. This extra scatter from the system can increase the scatterto-primary ratio (SPR) values by up to $31 \%$ for a typical DBT geometry (Sechopoulos et al. 2007b). In order to account for the scattered radiation beneath the breast phantom generated by the overlying system layers, an extra SPSF from the system $\left(S P S F_{\text {sys }}\right)$ was used. This was calculated using the same geometry, but in the absence of the uniform phantom (Figure 2(b)). Thus all the scattered radiation recorded within the image receptor was due to the different layers included in the X-ray mammography system. Due to the absence of phantom, the scattered radiation distribution changes more rapidly than when the phantom is present. Therefore, in order to calculate $S P S F s_{\text {sys }}$, primary incident angles $(\phi)$ from 0 to $35^{\circ}$ were used in steps of $5^{\circ}$.

Sechopoulos et al. (2007 $\underline{\mathrm{b}}$ ) also demonstrated the asymmetry of the SPSF as $\phi$ increases. Consequently, the spatial distributions of the SPSF were stored using polar 


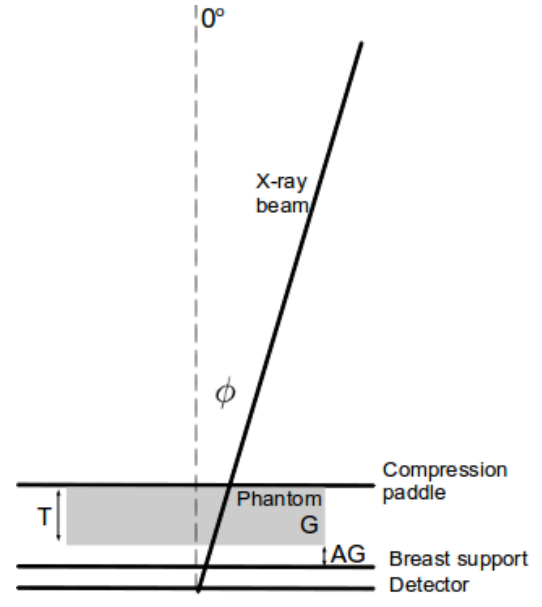

(a)

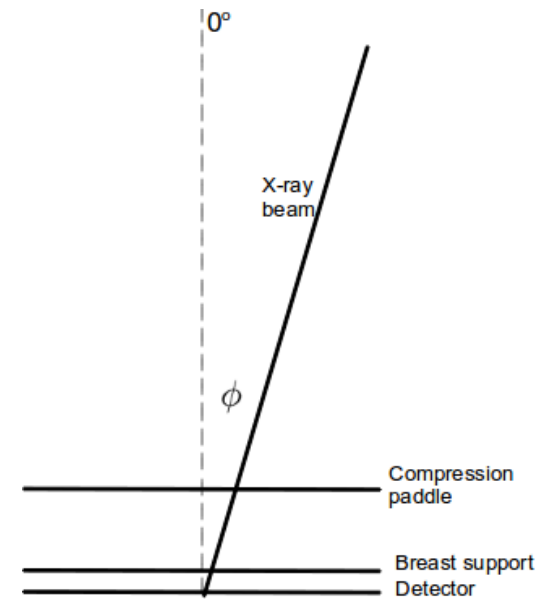

(b)

Figure 2. (a) Pencil beam geometry for a uniform phantom of glandularity $G$ and thickness $T$ used to calculate the $\mathrm{SPSF}_{b r e}$. The scatter response from the system $\mathrm{SPSF}_{\text {sys }}$ (without scattering material) was calculated as illustrated in (b).

coordinates $(r, \theta)$ for different radii $r$ and angles $\theta$ within the image plane in steps of 0.05 $m m(\Delta r)$ and $3^{\circ}(\Delta \theta)$ respectively. Intermediate $\mathrm{SPSF}_{\text {bre }}$ values have been calculated using linear interpolation under the assumption that the scatter field can be represented by a slowly varying function, devoid of fine structure. All calculated SPSF bre and $S P S F_{\text {sys }}$ were stored in look-up tables to construct the appropriate scatter kernel $K$ as described below.

\subsection{Scatter estimation (Section re-ordered)}

The total estimated scatter image $S_{T, \phi}(x, y)$ for a particular projection angle $\phi$ was estimated as a combination of the scatter image from primaries directed towards the breast $S_{b r e, \phi}(x, y)$ and the scatter from primaries directed outside the breast region $S_{\text {sys }, \phi}(x, y)$ :

$$
S_{T, \phi}(x, y)=S_{b r e, \phi}(x, y)+S_{s y s, \phi}(x, y) \quad[e V] .
$$

Following the methodology described in the literature to estimate scatter image using the kernel-based methods (Love \& Kruger 1987, Seibert \& Boone 1988, Boone \& Cooper 2000, Wu et al. 2009, Ducote \& Molloi 2010), $S_{b r e, \phi}(x, y)$ was calculated by convolving the corresponding primary image with an appropriate scatter kernel as follows:

$$
S_{b r e, \phi}(x, y)=\sum_{k_{1}=-a}^{a} \sum_{k_{2}=-b}^{b} P\left(k_{1}, k_{2}\right) K_{\phi, t, G, A G}\left(x-k_{1}, y-k_{2}\right) \quad[e V],
$$

where $P$ corresponds to the analytically calculated primary radiation image and $K_{\phi, t, G, A G}$ represents the appropriate scatter kernel. These, stored in look-up tables, were calculated using the SPSF for the realistic geometry shown in Figure 2(a). 
As demonstrated by several authors (Boone et al. 2000, Cooper et al. 2000, Sechopoulos et al. 2007b), scattered radiation is principally influenced by breast thickness $t$ and projection angle $\phi$. Although breast glandularity has a small effect on the scatter field, in this work $G$ represents the average glandularity of the entire breast phantom. The scatter kernel for a given glandulatiry $G$ was calculated as a linear combination of the scatter response for the $0 \%$ and $100 \%$ glandularity. Furthermore, the effect of the projected air gap $A G$ traversed by photons exiting the curved edge of the breast phantom before impinging on the breast support plate was also considered as suggested in Diaz et al. (2012) for highly idealised DBT geometries. This was first suggested by Diaz et al. (2012) for highly idealised DBT geometries. They found an improvement in scatter estimation when accounting for the air gap in the non-uniform thickness region observed in curved objects. This air gap is important to be considered because the scattered X-ray photons travel larger distances before impinging on the image receptor. Thus, at the skin edge, scattered photons project into air and no longer encounter the same scattering and attenuation path length experienced whilst traversing solid scattering material.

$S_{b r e, \phi}(x, y)$ was calculated assuming a parallel beam geometry, so that a fixed angle SPSF was used to calculate a single projection (at either $0,7.5$ or $25^{\circ}$ ) across the entire breast phantom area in order to speed up the process.

As described in the previous section, SPSFs were calculated using a geometry with a uniform thickness phantom, $\mathrm{SPSF}_{\text {bre }}$, and without uniform thickness phantoms $\left(\mathrm{SPSF}_{\text {sys }}\right)$. Therefore, a set of scatter kernels based on $\mathrm{SPSF}_{\text {bre }}$ was used within the breast phantom region (i.e. breast phantom shadow on image receptor) and a set based on $\mathrm{SPSF}_{\text {sys }}$ was used outside the confines of the breast phantom during the convolution process. Thus, $S_{\text {sys }, \phi}(x, y)$ was estimated after convolving the primary image at the image receptor $P(x, y)$ with a scatter kernel $K_{s y s, \phi, T}$ in the region outside the phantom.

$$
S_{s y s, \phi}(x, y)=\sum_{k_{1}=-a}^{a} \sum_{k_{2}=-b}^{b} P\left(k_{1}, k_{2}\right) K_{s y s, \phi, T}\left(x-k_{1}, y-k_{2}\right) \quad[e V] .
$$

$K_{s y s, \phi, T}$ depends only on the projection angle $(\phi)$ and the distance from the compression paddle to the breast support, i.e. breast thickness $T$, as the air gap between the breast support plate and image receptor is constant.

As previously described, $K_{s y s, \phi, T}$ was calculated in the absence of any scattering material (see Figure 2(b)). However in reality, the scattered radiation from the system is attenuated by the breast tissue. Therefore, the calculated $\mathrm{SPSF}_{\text {sys }}$ needs to be attenuated by the corresponding breast tissue thickness.

Figure 3 illustrates this process, with two cases of scattered X-ray photons generated within the compression paddle. In the case O-B, a photon is recorded within the image receptor at point $\left(\mathrm{x}_{1}{ }^{\prime}, \mathrm{y}_{1}{ }^{\prime}\right)$ after a scattering interaction within the paddle (whose primary X-ray photon corresponds to point (x,y) at the receptor) without being attenuated by any other tissue. Thus, Equation 4 is appropriate (no scattering material simulated). 
By contrast, the scattered photon $\mathrm{O}-\mathrm{A}$ is attenuated along the path it traverses to reach the image receptor at $\left(\mathrm{x}_{2}{ }^{\prime}, \mathrm{y}_{2}{ }^{\prime}\right)$.

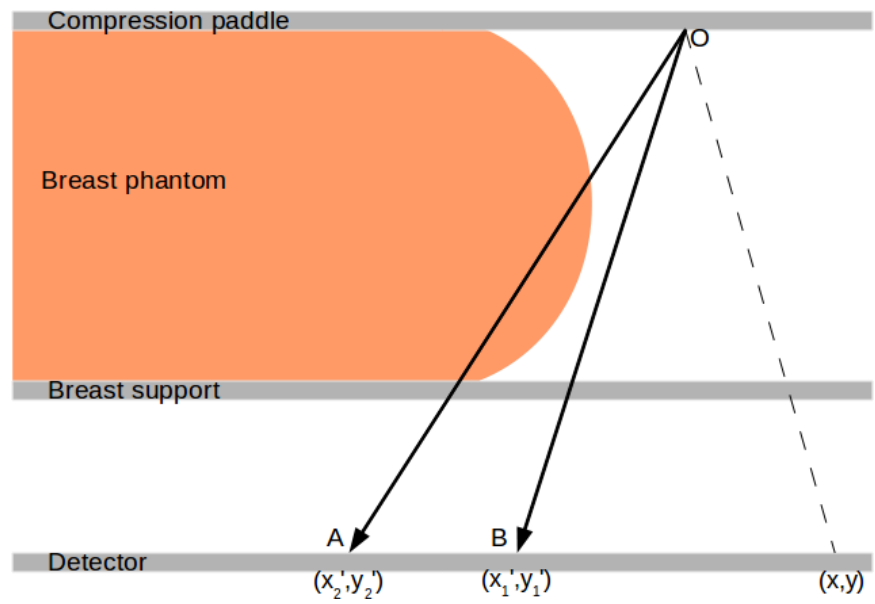

Figure 3. Two cases of scattered photons from the point $\mathrm{O}$ on the compression paddle, whose primary X-ray photon corresponds to point $(\mathrm{x}, \mathrm{y})$ at the image receptor. The ray $\mathrm{O}-\mathrm{B}$ shows an unattenuated photon which reaches the image receptor at $\mathrm{B}$. The photon scattered from the compression paddle following O-A path should be attenuated due to the path traversed within the breast phantom.

Thus, $K_{s y s, \phi, T}$ was corrected by a factor $\alpha$ to account for the attenuation path traversed by the photons from the compression paddle to the image receptor. This weight map $\alpha\left(x, y, x^{\prime}, y^{\prime}\right)$ compensates for the lack of scattering material outside the breast phantom $(\alpha=1)$ and for the attenuation due to the breast tissue $(\alpha<1)$ and was calculated as the ratio of the energy deposited within the image receptor with and without the breast phantom as follows:

$$
\alpha\left(x, y, x^{\prime}, y^{\prime}\right)=\frac{\sum_{E=E_{\min }}^{E_{\max }} \epsilon(E) \cdot w(E) \cdot E \cdot e^{\sum_{i}-\mu_{i}(E) \cdot t_{i}\left(x, y, x^{\prime}, y^{\prime}\right)} d E}{\sum_{E=E_{\min }}^{E_{\max }} \epsilon(E) \cdot w(E) \cdot E \cdot e^{\sum_{j}-\mu_{j}(E) \cdot t_{j}\left(x, y, x^{\prime}, y^{\prime}\right)} d E},
$$

where $\epsilon(E)$ and $w(E)$ correspond to the energy efficiency of the detector and the energy spectrum (normalised to an area of 1 ) observed after the compression paddle respectively. In this expression, the $\mu(E)$ are the linear attenuation coefficients and $t\left(x, y, x^{\prime}, y^{\prime}\right)$ are the distances from the compression paddle to the image receptor along the X-ray photon beam. The subscripts $i$ and $j$ correspond to the individual tissues found along the X-ray beam in the presence and absence of the breast phantom respectively. When breast tissue was found, $\mu(E)$ was calculated for the average glandularity of the breast phantom $G$ (Table 1 ). The distances $t\left(x, y, x^{\prime}, y^{\prime}\right)$ were calculated using ray tracing methodology (Siddon 1985).

The task of calculating the distances from every point in the compression paddle to each pixel in the detector is computationally expensive. Thus, in order to reduce computation time, the projection points in the compression paddle were separated by 3 $\mathrm{mm}$. Furthermore, only the pixels within the projected breast phantom shadow on the image receptor were evaluated. 
Once $\alpha\left(x, y, x^{\prime}, y^{\prime}\right)$ was calculated for each point within the compression paddle, $S_{s y s, \phi}$ was estimated as follows:

$$
S_{s y s, \phi}(x, y)=\sum_{k_{1}=-a}^{a} \sum_{k_{2}=-b}^{b} P\left(k_{1}, k_{2}\right) K_{s y s, \phi, T}\left(x-k_{1}, y-k_{2}\right) \alpha\left(x, y, x^{\prime}, y^{\prime}\right) .
$$

To account for the primary X-ray incident angle at each evaluated point in the compression paddle, the X-ray incident angle and the direction of the primary X-ray photons were taken into account. For a given point in the compression paddle (outside the breast phantom area), the closest simulated incident angle (previously calculated from 0 to $35^{\circ}$ in steps of $5^{\circ}$ ) was used.

\subsection{Relative scatter error map}

In order to validate the kernel-based methodology, scattered radiation images from the proposed method $S_{T, \phi}(x, y)$ were compared with scatter images from direct MC simulations $S_{M C}$, which represents the ground truth. Maps of scatter relative error for each pixel $\epsilon_{S}$, were created as:

$$
\epsilon_{S}(x, y)=\frac{S_{M C}(x, y)-S_{T, \phi}(x, y)}{S_{M C}(x, y)} \times 100 \quad[\%] .
$$

Furthermore, scatter fields calculated using the proposed scatter kernels $\left(K_{\phi, t, G, A G}\right)$, which account for the air gap between breast and breast support plate, and the conventional thickness-dependent scatter kernels described in the literature $\left(K_{\phi, t, G}\right)$ were compared. For the purposes of this work, discrepancies of less than $10 \%$ for $\epsilon_{S}(x, y)$ are desirable. As the scattered radiation is typically lower than the primary, the error in the total image (primary+scatter) is expected to be smaller than this target of $10 \%$. The average SPR values observed for the 22,50 and $77 \mathrm{~mm}$ thick phantoms were $0.3,0.5$ and 1.0 respectively. Therefore, there will be a total error of $2.3,3.3$ and $5.0 \%$ respectively in the total image (primary+scatter) for a $10 \%$ error in the scatter image.

\subsection{Validation of SPSFs}

SPSFs from this work were validated against data published by Sechopoulos et al. (2007b), using the beam qualities and geometries given in Table 2.

Table 2. Configuration of the experiments used to validate the SPSF with Sechopoulos et al. (2007b) using an idealised pencil beam geometry.

\begin{tabular}{ccccc} 
Exp & Energy Spectrum $(\mathrm{keV})$ & Phantom Thickness $(\mathrm{cm})$ & Glandularity $(\%)$ & Air gap $(\mathrm{mm})$ \\
\hline $\mathrm{A}$ & $26 \mathrm{Mo} / \mathrm{Mo}$ & 5 & $0,50,100 \&$ water & 10 \\
$\mathrm{~B}$ & $26 \mathrm{Mo} / \mathrm{Mo}, 32 \mathrm{Rh} / \mathrm{Rh}$ & 5 & 50 & 10 \\
$\mathrm{C}$ & $26 \mathrm{Mo} / \mathrm{Mo}$ & 4 & 50 & $0,10,20 \& 30$ \\
$\mathrm{D}$ & $26 \mathrm{Mo} / \mathrm{Mo}$ & $2,4,6 \& 8$ & 50 & 10
\end{tabular}

The validation process used an idealised pencil beam geometry as shown in Figure 4(a). Results of this validation are shown in the next section. 


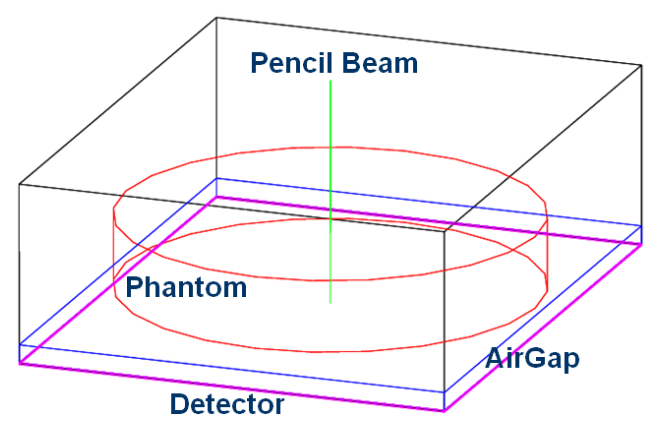

(a) Pencil beam geometry

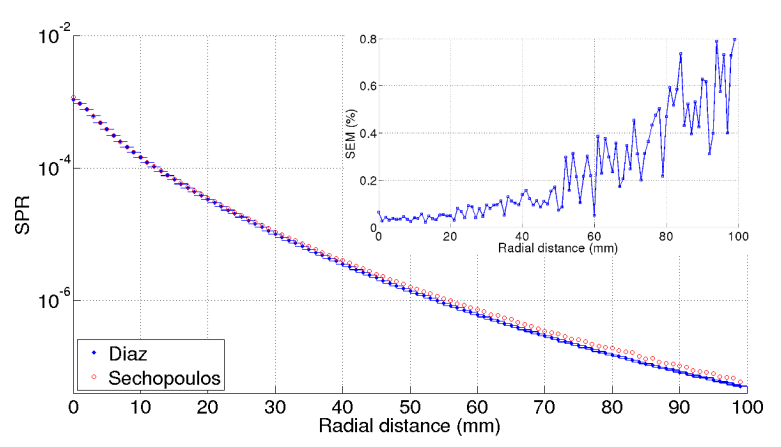

(b) SPSF $2 \mathrm{~cm}$ thick uniform phantom

Figure 4. The pencil beam geometry used for the idealised geometry is shown in (a). (b) shows the SPSFs from this work (Diaz) and Sechopoulos et al. (2007b) when using a D configuration (see Table 2) for a phantom thickness of $2 \mathrm{~cm}(\mathrm{G}=50 \%)$. The SEM associated to the MC simulations of this work as a function of distance is shown in the top right corner.

\section{Results}

\subsection{Primary calculation}

The average error found between the analytically calculated primary $P(x, y)$ and primary from direct $\mathrm{MC}$ simulations was $0.2 \%$ and the largest disagreement was $0.5 \%$, illustrating that the difference using a single or several points within the pixel area was small.

\subsection{SPSFs validations}

A sample SPSF from this validation process is shown in Figure 4(b). In this figure, it is observed that the SEM associated with the MC simulations increases with radial distance as fewer photons reach the image receptor. A quantitative comparison between the SPSFs from this work and Sechopoulos et al. (2007b) is presented in Table 3. The SPR values were calculated by integrating the area under each SPSF curve using a circular field of view of radius $100 \mathrm{~mm}$. The average SEMs (in \%) associated to the results for this work are shown in brackets. Sechopoulos et al. (2007b) described their SEM errors as $0.4 \%$ and $1.6 \%$ for the $5 \mathrm{~cm}$ and $8 \mathrm{~cm}$ phantoms respectively $(50 \%$ glandularity and $27 \mathrm{kVp}$ ).

The largest discrepancy found was $4.0 \%$ for $100 \%$ glandular breast tissue compared with the minimum difference observed of $0.3 \%$ for a uniform phantom filled in with water. In this work, the breast tissue composition from Hammerstein et al. (1979) was employed. However, this discrepancy may be due to different tissue composition assumed as good agreement was observed when simulating water. Despite these small discrepancies (maximum of 4\%), the close agreement of the results from this work and the literature suggest that the SPSF generated here can be used with confidence to generate scatter kernels when using a more realistic geometry. 
Table 3. Comparison of SPR values for Sechopoulos et al. (2007b) and results from this work using a circular field of view of radius $100 \mathrm{~mm}$ for the setups described in Table 2. SEMs (in \%) for this work are shown in brackets. Maximum and minimum differences are highlighted in bold.

\begin{tabular}{|c|c|c|c|c|}
\hline \multirow{2}{*}{ Exp. label } & & \multicolumn{2}{|c|}{ Area under the curve (SPR) } & \multirow{2}{*}{ Difference (\%) } \\
\hline & & Sechopoulos & Diaz (SEM,\%) & \\
\hline \multirow{4}{*}{ A } & $\mathrm{G}=0 \%$ & $5.33 \times 10^{-1}$ & $5.23 \times 10^{-1}(0.2)$ & 1.9 \\
\hline & $\mathrm{G}=50 \%$ & $5.57 \times 10^{-1}$ & $5.40 \times 10^{-1}(0.4)$ & 3.0 \\
\hline & $\mathrm{G}=100 \%$ & $5.92 \times 10^{-1}$ & $5.68 \times 10^{-1}(0.9)$ & 4.0 \\
\hline & water & $5.91 \times 10^{-1}$ & $5.89 \times 10^{-1}(0.6)$ & 0.3 \\
\hline \multirow{2}{*}{ B } & 26MoMo & $5.57 \times 10^{-1}$ & $5.40 \times 10^{-1}(0.4)$ & 3.0 \\
\hline & $32 \mathrm{RhRh}$ & $5.70 \times 10^{-1}$ & $5.55 \times 10^{-1}(0.2)$ & 2.6 \\
\hline \multirow{4}{*}{$\mathrm{C}$} & $\mathrm{AG}=0 \mathrm{~mm}$ & $4.57 \times 10^{-1}$ & $4.43 \times 10^{-1}(0.8)$ & 3.0 \\
\hline & $\mathrm{AG}=10 \mathrm{~mm}$ & $4.55 \times 10^{-1}$ & $4.41 \times 10^{-1}(0.3)$ & 3.0 \\
\hline & $\mathrm{AG}=20 \mathrm{~mm}$ & $4.48 \times 10^{-1}$ & $4.36 \times 10^{-1}(0.2)$ & 2.7 \\
\hline & $\mathrm{AG}=30 \mathrm{~mm}$ & $4.37 \times 10^{-1}$ & $4.26 \times 10^{-1}(0.2)$ & 2.5 \\
\hline \multirow{4}{*}{$\mathrm{D}$} & $\mathrm{T}=2 \mathrm{~cm}$ & $2.44 \times 10^{-1}$ & $2.39 \times 10^{-1}(0.2)$ & 2.2 \\
\hline & $\mathrm{T}=4 \mathrm{~cm}$ & $4.55 \times 10^{-1}$ & $4.41 \times 10^{-1}(0.4)$ & 3.0 \\
\hline & $\mathrm{T}=6 \mathrm{~cm}$ & $6.63 \times 10^{-1}$ & $6.40 \times 10^{-1}(0.5)$ & 3.5 \\
\hline & $\mathrm{T}=8 \mathrm{~cm}$ & $8.72 \times 10^{-1}$ & $8.43 \times 10^{-1}(0.8)$ & 3.3 \\
\hline
\end{tabular}

\subsection{Comparison of proposed scatter kernels with conventional scatter kernels}

The proposed kernel-based scatter field estimation method was applied to the $50 \mathrm{~mm}$ thick breast phantom (Table 1). Figure 5 illustrates the associated error maps. A bipolar colour map has been used to illustrate the change in error polarity across the breast phantom projection. Results using the conventional kernel-based approach (top row), which is confined to thickness dependent scatter kernels $\left(K_{\phi, t, G}\right)$, were compared with the scatter images when using the approach suggested in this work (bottom row), which account for the air gap between the breast phantom and breast support plate $\left(K_{\phi, t, G, A G}\right)$. It is observed that the conventional thickness-dependent scatter kernel starts to show a large overestimation of scatter (dark blue area) as the projection angle increases (note the X-ray tube tilts towards the bottom of the page). This is because the air gap between the lower curvature of the breast and the breast support plate increases as well and this effect is not taken into account when using the conventional thickness-dependent scatter kernels. However, when the air gap is taken into account as suggested in this paper, these errors are largely reduced as observed in the bottom row of Figure 5.

In order to understand the magnitude of the errors observed in Figure 5, histograms of the errors for each of the images are shown in Figure 6. In this figure, the histogram of the errors when using conventional thickness-dependent scatter kernels (left column) can be compared with the histogram of the errors from the scatter kernels suggested in this work (right column), where a thickness and air gap dependent scatter kernel was used. In all the histograms shown, it is observed that the tail of the error distributions are much reduced when accounting for the air gap in the scatter kernel. Errors as large as $30 \%$ are reduced to no more than $10 \%$. This has shown a reduction in the total image (primary+scatter) error from $20 \%$ to $6 \%$ for the $25^{\circ}$ case. Table 4 gives the fraction of the breast phantom area for which the errors are equal to or less than the target of 


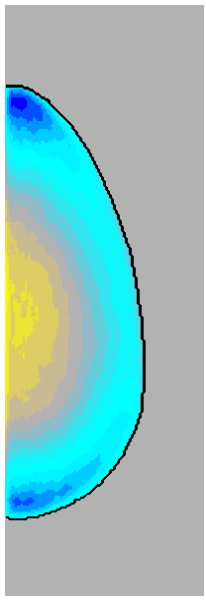

(a) $0^{\circ}$

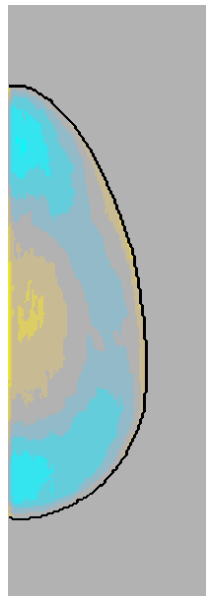

(d) $0^{\circ}$

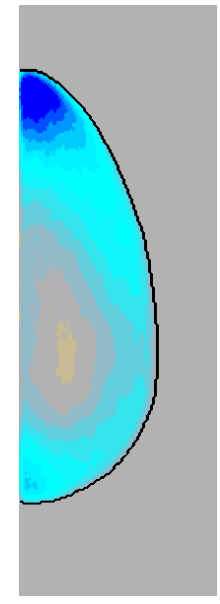

(b) $7.5^{\circ}$

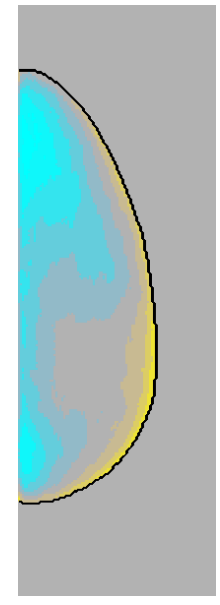

(e) $7.5^{\circ}$

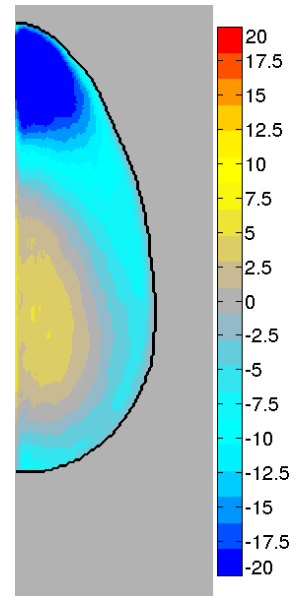

(c) $25^{\circ}$

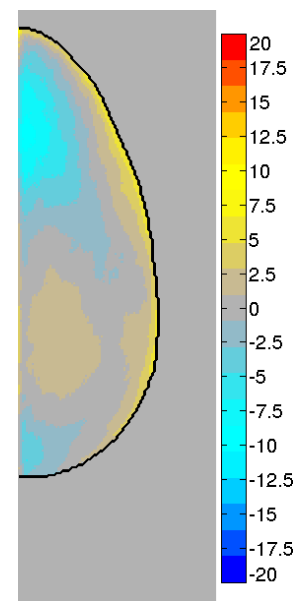

(f) $25^{\circ}$

Figure 5. Scatter relative error map $\epsilon_{S}(x, y)$ between scatter estimates using scatter kernels and MC estimates, observed for the $50 \mathrm{~mm}$ thick breast phantoms $(\mathrm{G}=20 \%)$ and projection angles of $0,7.5$ and $25^{\circ}$. Top row illustrates results using the conventional thickness-dependent scatter kernels $\left(K_{\phi, t, G}\right)$. The results using the approach proposed in this work $\left(K_{\phi, t, G, A G}\right)$ are illustrated in the bottom row. Each colour map shows errors between $-20 \%$ and $20 \%$. The edge of the breast phantom is highlighted in black.

$10 \%$ for the $50 \mathrm{~mm}$ breast phantom. As the tube angle increases, the effects of the edge curvature increase leading to greater difference between the two approaches.

Similarly, the estimated scatter fields generated using both approaches were investigated for the 22 and $77 \mathrm{~mm}$ thick anthropomorphic breast phantoms. The percentages of the projected breast area with errors equal to or less than $10 \%$ (target) for both scatter kernel approaches are also presented in Table 4. There was only a small improvement observed when accounting for the air gap for the $22 \mathrm{~mm}$ thick breast phantom. This is attributed to the smaller air gaps between the curved region of the breast phantom and the breast support plate for such a thin phantom. In contrast, the 


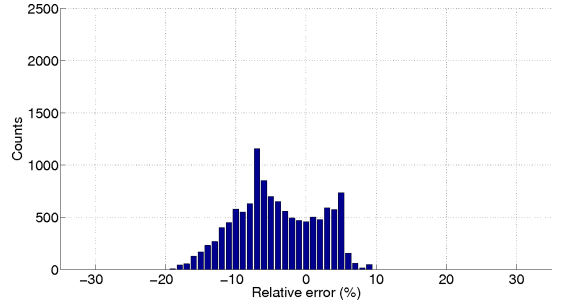

(a) Conventional scatter kernel

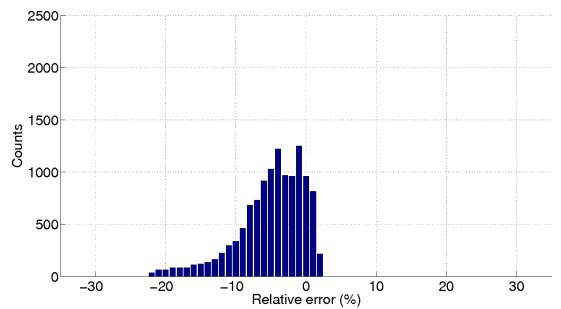

(c) Conventional scatter kernel

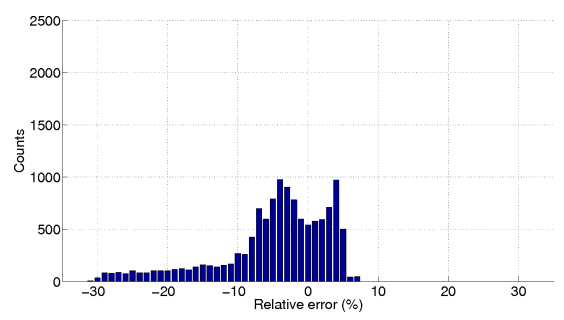

(e) Conventional scatter kernel

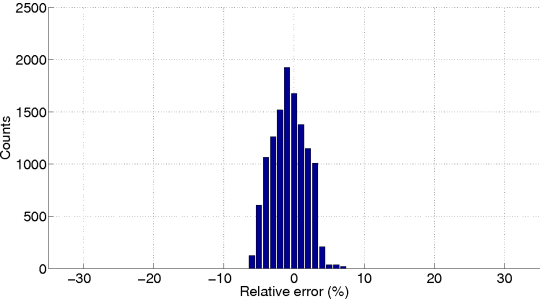

(b) Proposed scatter kernel

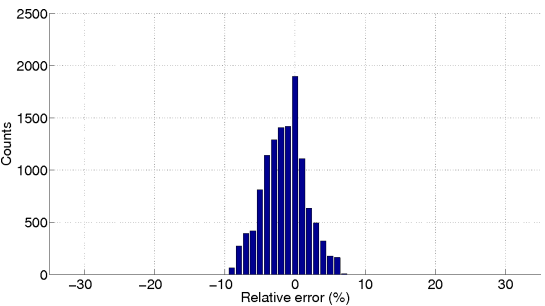

(d) Proposed scatter kernel

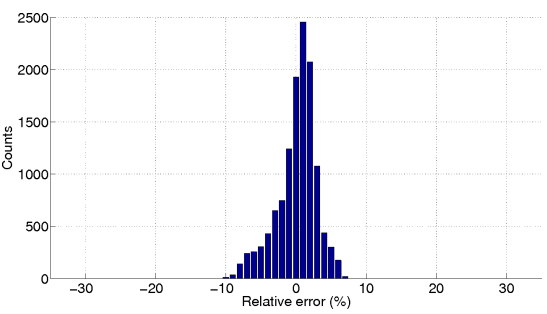

(f) Proposed scatter kernel

Figure 6. Histograms of the scatter relative error maps $\epsilon_{S}(x, y)$ observed for the 50 $\mathrm{mm}$ thick breast phantoms $(\mathrm{G}=20 \%)$ shown in Figure 5. Top, middle and bottom row illustrate results for $0^{\circ}, 7.5^{\circ}$ and $25^{\circ}$. The left column shows the histogram for the conventional approach of breast thickness dependent scatter kernels, whereas the right column shows the histogram using the approach proposed in this work.

Table 4. Percentage of the projected breast phantom area with errors equal or less than $10 \%$ (target) when using the conventional thickness-dependent scatter kernels and the scatter kernels proposed in this work. Results for the 22, 50 and $77 \mathrm{~mm}$ thick breast phantoms and the projection angles studied are shown. Last row gives the difference between the methodologies.

\begin{tabular}{c|ccc|ccc|ccc} 
Breast phantom thick. & \multicolumn{3}{|c}{$22 \mathrm{~mm}$} & \multicolumn{3}{c}{$50 \mathrm{~mm}$} & \multicolumn{3}{c}{$77 \mathrm{~mm}$} \\
Method & $0^{\circ}$ & $7.5^{\circ}$ & $25^{\circ}$ & $0^{\circ}$ & $7.5^{\circ}$ & $25^{\circ}$ & $0^{\circ}$ & $7.5^{\circ}$ & $25^{\circ}$ \\
\hline Conventional kernel & $100 \%$ & $98 \%$ & $96 \%$ & $81 \%$ & $85 \%$ & $80 \%$ & $73 \%$ & $73 \%$ & $74 \%$ \\
Proposed kernel & $100 \%$ & $100 \%$ & $100 \%$ & $100 \%$ & $100 \%$ & $100 \%$ & $100 \%$ & $91 \%$ & $88 \%$ \\
Difference & $0 \%$ & $2 \%$ & $4 \%$ & $19 \%$ & $15 \%$ & $20 \%$ & $27 \%$ & $18 \%$ & $14 \%$
\end{tabular}

1 improvements observed when accounting for the air gap in the $77 \mathrm{~mm}$ breast phantom were more than $25 \%$ for $0^{\circ}$, illustrating the importance of accounting for the air gap for accurate scatter field estimation.

Table 5 gives the percentage area of the projected breast phantom with errors equal to or less than various error thresholds (5, 10, 12 and 15\%) when comparing the 
proposed scatter field estimator approach with direct MC simulations (ground truth data). Furthermore, the average error (ave. error) and average error of absolute values (ave. error (abs)) are also shown for each of the error maps. In all the studied cases, the proposed scatter kernels estimates scatter fields within the $10 \%$ error target for a minimum of $88 \%$ of the projected breast phantom area. The average error also confirms the good overall performance of the proposed approach, as a maximum of $-3 \%$ error (minus sign denotes overestimation) was observed.

Table 5. Percentage of the projected breast phantom area with errors equal or less than various thresholds when using the scatter kernels proposed in this work.

\begin{tabular}{|c|c|c|c|c|c|c|c|c|}
\hline Thickness & Angle & $\begin{array}{c}\text { SPR } \\
0.3\end{array}$ & $\begin{array}{c}\text { Max. thres. } \\
\text { error } 5 \%\end{array}$ & $\begin{array}{c}\text { Max. thres. } \\
\text { error } 10 \%\end{array}$ & $\begin{array}{l}\text { Max. thres. } \\
\text { error } 12 \%\end{array}$ & $\begin{array}{c}\text { Max. thres. } \\
\text { error } 15 \%\end{array}$ & $\begin{array}{l}\text { Ave. } \\
\text { error }\end{array}$ & $\begin{array}{c}\text { Ave. } \\
\text { error (abs) }\end{array}$ \\
\hline \multirow{3}{*}{$22 \mathrm{~mm}$} & $0^{O}$ & 0.3 & $98 \%$ & $100 \%$ & $100 \%$ & $100 \%$ & $-1 \%$ & $2 \%$ \\
\hline & $7.5^{\circ}$ & 0.3 & $93 \%$ & $100 \%$ & $100 \%$ & $100 \%$ & $-2 \%$ & $2 \%$ \\
\hline & $25^{\circ}$ & 0.5 & $96 \%$ & $100 \%$ & $100 \%$ & $100 \%$ & $-2 \%$ & $2 \%$ \\
\hline \multirow[b]{2}{*}{$50 \mathrm{~mm}$} & $0^{O}$ & 0.5 & $93 \%$ & $100 \%$ & $100 \%$ & $100 \%$ & $-1 \%$ & $2 \%$ \\
\hline & $7.5^{\circ}$ & 0.5 & $82 \%$ & $100 \%$ & $100 \%$ & $100 \%$ & $-2 \%$ & $3 \%$ \\
\hline \multirow[t]{2}{*}{$77 \mathrm{~mm}$} & $7.5^{\circ}$ & 0.9 & $68 \%$ & $91 \%$ & $96 \%$ & $99 \%$ & $-3 \%$ & $5 \%$ \\
\hline & $25^{\circ}$ & 1.1 & $56 \%$ & $88 \%$ & $94 \%$ & $98 \%$ & $0 \%$ & $5 \%$ \\
\hline
\end{tabular}

\section{Discussion and conclusions}

One of the main disadvantages of most DBT systems is the large scattered radiation fraction observed at the image receptor. For system modelling and optimisation or scatter correction/removal, a good understanding of the scatter signal for each of the DBT projections is required. Direct MC simulations are often used to study scattered radiation. However, it is well known that these can be computationally expensive so that several tens of hours might be needed to estimate an appropriate scatter field for each DBT projection. As a consequence, a faster approach to estimate scattered radiation fields is desirable. The kernel-based approach has previously been used to calculate the scatter field in planar 2D X-ray mammography, estimating the scatter by convolving the primary image with a scatter kernel, which varies across the image based on the thickness of the breast.

One of the principal limitations of prior work based on this approach has been the highly idealised objects and geometries used. In this work more realistic phantom geometries have been integrated with a detailed geometrical model of a particular DBT system. The resulting thickness-dependent scatter kernels were studied for a series of anthropomorphic breast phantoms suggesting that kernels just based on thickness overestimated the scattered radiation in certain regions of the breast phantom. This effect was exacerbated by increasing projection angle. As described previously for idealised DBT geometry (Diaz et al. 2012), this overestimation of scattered radiation was mainly due to the air gap between the lower curved surface of the object and the breast support plate. 
In this work, the kernel-based approach was adapted to account for this air gap, demonstrating an improvement in the scatter signal estimation when using anthropomorphic breast phantoms in realistic DBT geometries, which include the compression paddle and breast support plate. In this more realistic scenario, the scattered radiation from the compression paddle and breast support plate was taken into account for the first time. Results showed that the kernel-based approach suggested in this work, can estimate the scattered radiation to within a 10\% error across most of the breast area when compared with direct MC simulations. Furthermore, even with no attempts to optimise the computation time, this was reduced from approximately 10 hours (direct MC simulations) to less than 1.5 hours per projection when using the proposed kernel-based approach §. Alternatively, MC codes can also be optimised to reduce their computational time. However, the kernel-based methodology represents a more flexible approach as the scatter kernels have been previously calculated.

The scatter kernel approach suggested here was tested using three representative anthropomorphic breast phantoms developed by Bakic et al. (2011). It was noticed that the scattered radiation was highly dependent on the curvature at the breast edge, which has been previously associated to the breast size and compression force (AlonzoProulx et al. 2008). Furthermore, it has been suggested (Morgan 2012) that breast composition will have an important role in the breast curvature as denser breasts will have more symmetrical curvature than adipose breasts. For this reason, it would be desirable to quantify in future the actual compressed breast curvatures to have a better understanding of how critical may this problem be.

The proposed kernel-based method suggests calculating the SPSFs using narrow pencil beams in homogeneous phantoms. Thus the scatter response can be applied in breast models with similar densities (adipose tissue, glandular tissue). However, if this methodology is applied in a different environment where high density tissues (e.g. bones) are interlaced with low density tissues, the SPSFs would need to be modified to account for the assymety observed when X-ray photons are transmitted through tissues of very different densities (i.e. heterogenous). These modifications would probably increase the computational time, making this method unsuitable for fast calculation.

\section{Acknowledgement}

This work was part of the OPTIMAM project and was supported by Cancer Research UK \& EPSRC Cancer Imaging Programme in Surrey, in association with the MRC and Department of Health (England). The authors would like to thank Baorui Ren from Hologic, Inc for providing helpful information about the system geometry and also Ms Grainne Morgan from the Jarvis Breast Screening Centre (Guildford) for useful discussions about compressed breast shapes. Furthermore, O Diaz would like to thank Dr Mary Yip from University College London for useful discussion and proof reading.

$\S$ Using a 2.2GHz Intel Quad-Core E5520 processor. 
1

\section{References}

Agostinelli S et al 2003 Geant4 - a simulation toolkit Nuclear Instruments and Methods in Physics Research 506(3), 250-303.

Allison J et al 2006 Geant4 developments and applications IEEE Transactions on Nuclear Science 53(1), 270-278.

Alonzo-Proulx O et al 2008 Effect of Tissue Thickness Variation in Volumetric Breast Density Estimation in E.A. Krupinski, ed., 'International Workshop on Digital Mammography' Vol. 5116 of LNCS Springer Berlin Heidelberg pp. 659-666.

Baker J \& Lo J 2011 Breast Tomosynthesis: State-of-the-Art and Review of the Literature Academic Radiology 18, 1298-1310.

Bakic P, Zhang C \& Maidment A 2011 Development and characterization of an antropomorphic breast software phantom based upon region-growing algorithm Medical Physics 38(6), 3165-3176.

Boone J \& Cooper V 2000 Scatter/primary in mammography: Monte Carlo validation Medical Physics 27(8), 1818-1831.

Boone J, Fewell T \& Jennings J 1997 Molybdenum, rhodium and tungsten anode spectral models using interpolating polynomials with applications to mammography Medical Physics 24(2), 18631874.

Boone J et al 2000 Scatter/primary in mammography: Comprehensive results Medical Physics $\mathbf{2 7}(10), 2408-2416$.

Boone J et al. 2002 Grid and slot scan scatter reduction in mammography: Comparison by using Monte Carlo techquines Radiology 222, 519-527.

Chen Z \& Ning R 2003 Why should breast tumour detection go three dimensional? Physics in Medicine and Biology 48, 2217-2228.

Cooper V et al 2000 An edge spread technique for measurement of the scatter-to-primary ratio in mammorgaphy Medical Physics 27(5), 845-853.

Cunha D, Tomal A \& Poletti M 2010 Evaluation of scatter-to-primary ratio, grid performance and normalized average glandular dose in mammography by Monte Carlo simulation including interference and energy broadening effects Physics in Medicine and Biology 55, 4335-4359.

Dance D \& Day G 1984 The computation of scatter in mammography by Monte Carlo methods Physics in Medicine and Biology 29(3), 237-247.

Diaz O 2013 Scattered radiation in projection X-ray mammography and digital breast tomosynthesis $\mathrm{PhD}$ thesis University of Surrey.

Diaz O et al 2012 A fast scatter field estimator for Digital Breast Tomosynthesis in 'SPIE Medical Imaging' Vol. 8313831305.

Dobbins J \& Godfrey D 2003 Digital x-ray tomosynthesis: current state of the art and clinical potential Physics in Medicine and Biology 48, R65-R106.

Ducote J \& Molloi S 2010 Scatter correction in digital mammography based on image deconvolution Physics in Medicine and Biology 55, 1295-1309.

Ferlay $\mathrm{J}$ et al 2010 Estimates of worldwide burden of cancer in 2008: GLOBOCAN 2008 International Journal of Cancer 127, 2893-2917.

Hammerstein G et al 1979 Absorbed radiation dose in mammography Radiology 130, 485-491.

ICRU 1992 Photon, electron, proton and neutron interaction data for body tissues Technical Report 46 International Commission on Radiation Units and Measurements.

Kolitsi Z et al 1992 A multiple projection method for digital tomosynthesis Medical Physics 19(4), 10451050.

Love L \& Kruger R 1987 Scatter estimation for a digital radiographic system using convolution filtering Medical Physics 14(2), 178-185.

Morgan G 2012 personal communication.

Niklason L T et al 1997 Digital Tomosynthesis in Breast Imaging Radiology 205, 399-406.

Rashidnasab A et al 2013 Simulation and assessment of realistic breast lesions using fractal growth 
models Physics in Medicine and Biology 58(16), 5613-5627.

Sechopoulos I 2013 A review of breast tomosynthesis. Part I. The image acquisition process Medical Physics 40(1), 014301-1.

Sechopoulos I et al 2007 $\underline{b}$ Scatter radiation in digital tomosynthesis of the breast Medical Physics 34(2), 564-576.

Seibert J \& Boone J 1988 X-ray scatter removal by deconvolution Medical Physics 15(4), 567-575.

Siddon R 1985 Fast calculation of the exact radiological path for a three-dimensional CT array Medical Physics 12(2), 252-255.

Skaane $\mathrm{P}$ et al 2013 Comparison of digital mammography alone and digital mammography plus tomosynthesis in a population-based screening program Radiology 267, 652-660.

Wallis M et al 2012 Two-view and single-view tomosynthesis versus full-field digital mammography : high-resolution X-ray imaging observer study Radiology 262, 788-796.

Wu G et al 2009 Evaluation of scatter effects on image quality for breast tomosynthesis Medical Physics 36(10), 4425-4432. 